This item was submitted to Loughborough's Research Repository by the author.

Items in Figshare are protected by copyright, with all rights reserved, unless otherwise indicated.

\title{
Improvements of material removal in cortical bone via impact cutting method
}

PLEASE CITE THE PUBLISHED VERSION

https://doi.org/10.1016/j.jmbbm.2020.103791

PUBLISHER

Elsevier BV

VERSION

AM (Accepted Manuscript)

PUBLISHER STATEMENT

This paper was accepted for publication in the journal Journal of the Mechanical Behavior of Biomedical Materials and the definitive published version is available at https://doi.org/10.1016/j.jmbbm.2020.103791.

\section{LICENCE}

CC BY-NC-ND 4.0

\section{REPOSITORY RECORD}

Bai, Wei, Liming Shu, Ronglei Sun, Jianfeng Xu, Vadim Silberschmidt, and Naohiko Sugita. 2020. "Improvements of Material Removal in Cortical Bone via Impact Cutting Method". figshare. https://hdl.handle.net/2134/12196074.v1. 


\title{
Improvements of material removal in cortical bone via impact cutting method
}

\author{
Wei Bai ${ }^{\text {a,b }}$, Liming Shu ${ }^{\text {b }}$, Ronglei Sun ${ }^{\text {a }}$, Jianfeng $\mathrm{Xu}^{\text {a, }}{ }^{* *}$, Vadim V. Silberschmidt \\ c, Naohiko Sugita ${ }^{\text {,** }}$ \\ ${ }^{\text {a }}$ State Key Lab of Digital Manufacturing Equipment and Technology, Huazhong University of Science and Technology, Wuhan, 430074, China ${ }^{\text {b }}$ \\ Department of Mechanical Engineering, School of Engineering, The University of Tokyo, 7-3-1 Hongo, Bunkyo-ku, Tokyo, 1138656, Japan \\ ${ }^{\mathrm{c}}$ Wolfson School of Mechanical, Electrical and Manufacturing Engineering, Loughborough University, Leicestershire, LE11 3TU, UK
}

\begin{abstract}
A B S T R A C T
Bone cutting with high efficiency as well as low levels of forces and damage has a great significance for orthopaedic surgeries. Due to the brittleness and anisotropy of cortical bone, a conventional cutting process can cause irregular crack propagation and fractured bone chip, affecting the tissue removal process and postoperative recovery. In this paper, a high-frequency impact cutting method is investigated, and its effect on fracture propagation, chip formation and cutting forces is studied for orthogonal cutting. Experimental results show that cracks are deflected by cement lines in conventional cutting, forming fractured blocks or split chips. In impact cutting, the cutting-induced fractures expand along a main shear direction, generating small pieces of triangular segmented chips. Cutting forces are significantly reduced with vibration-induced impacts; especially, the main cutting force is nearly $70 \%$ lower than that in the conventional cutting. The main reason for this is much higher strain rates in high-frequency impact cutting than in a conventional process, and direct penetration of fractures across the osteonal matrix without deflections along the cement lines. This results in a straighter path along the main shear plane and totally different chip morphology; so, a lower consumption of cutting energy in the main shear direction reduces the macroscopic cutting force. The results of this study have an important theoretical and practical value for revealing the mechanism of impact cutting, improving the efficiency of osteotomy and supporting the innovation in bone surgical instruments.
\end{abstract}

\author{
Cortical bone \\ Impact cutting \\ Fracture propagation \\ Chip morphology \\ Cutting force \\ Fracture toughness
}

\section{Introduction}

Bone cutting is an important procedure in surgical operations, covering bone drilling, grinding, sawing etc. Due to a quasi-brittle character and anisotropy of cortical bone, various scenarios of crack propagation and deep injury of tissues are caused, affecting the orthopaedic operation and postoperative recovery. Thus, investigation of the mechanism of bone cutting is crucial for improvement of the bone-tissue removal in surgery.

Orthogonal bone cutting was initially investigated by Jacobs et al. (1974), who observed the chip types with microscopic and SEM photography. Sugita and Mitsuishi (2009) analyzed the characteristics of fracture and crack propagation in cortical bone in relation to its microstructure. Recently, Liao and Axinte (2016) suggested a fracture mechanism based on a chip-formation model in an orthogonal bone-cutting process and presented a model of transition between three cutting modes with an increase in the depth of cut. Feldmann et al. (2017) measured cutting forces, temperature and chip formation for cortical bone in real time in orthogonal cutting, establishing relationships between the depth of cut, cutting forces and temperature. Bai et al. (2020) revealed the mechanism of chip formation in the orthogonal cutting of the cortical bone, demonstrating importance of the correlation between the chip morphologies, the depth of cut and the microstructure and sub-microstructure of cortical bone. Scholars found that various chip morphologies were produced with different depths-of-cut, and severe crack propagation, fractured chips, large cutting forces were generated at their higher levels, often used in surgical procedures. Therefore, development of innovative tools and novel bone-cutting techniques is necessary to improve material-removal efficiency, reduce tissue damage and decrease machining forces.

\footnotetext{
* Corresponding author.

$*$ Corresponding author.

E-mail addresses: wbai@hust.edu.cn (W. Bai), jfxu@hust.edu.cn (J. Xu), sugi@mfg.t.u-tokyo.ac.jp (N. Sugita).
} 

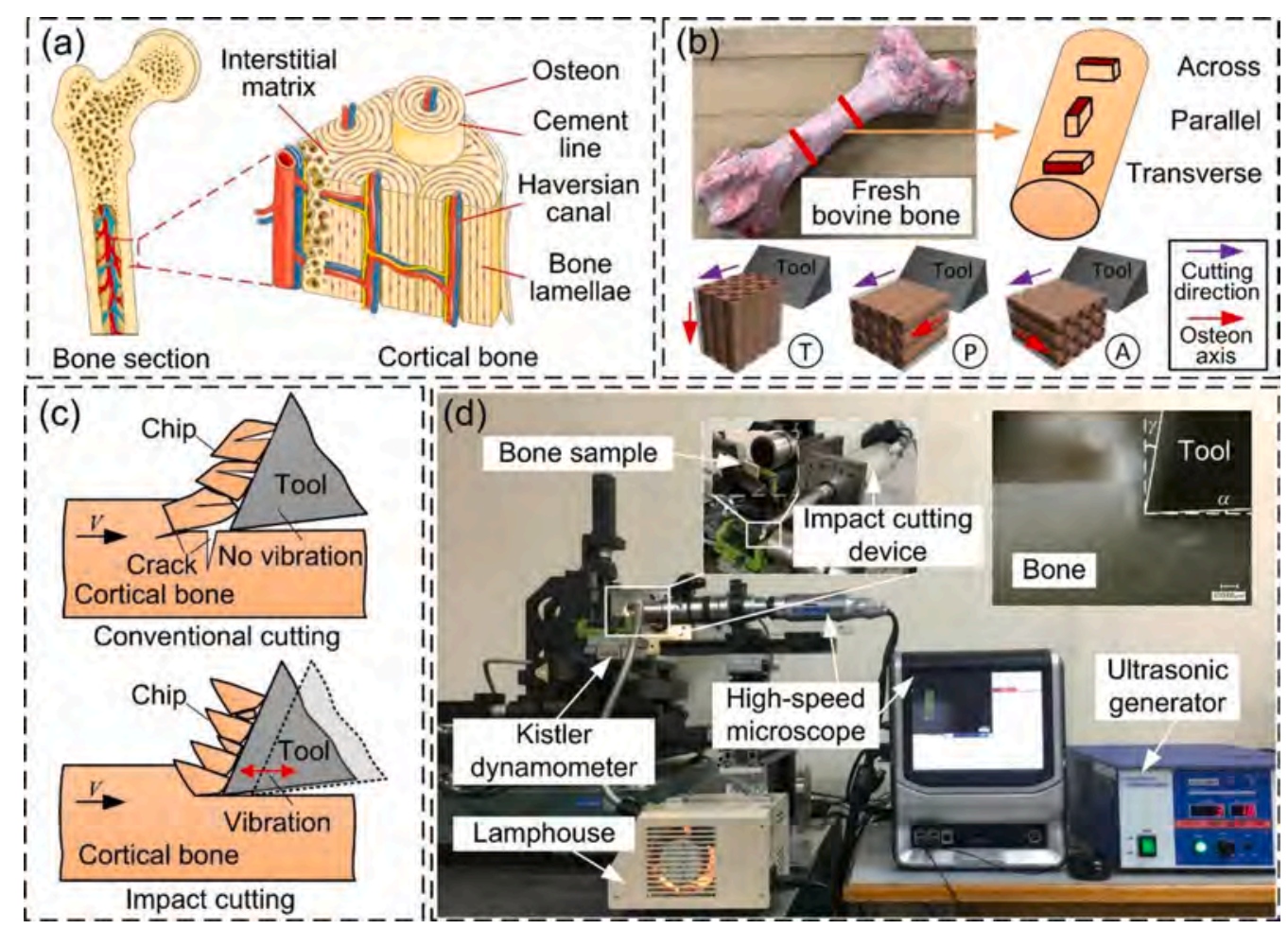

Fig. 1. (a) Characteristics of cortical bone; (b) sample preparation; (c) schematic diagram of impact cutting; (d) experimental setup of high-frequency impact cutting system. Fig. 1(a) was adapted from Servier Medical Art (https://smart.servier.com). (The across direction coincides with the radial direction in the bone.)

Tools for reduction of cutting-induced forces and tissue damage were a focus of studies by many researchers. A multi-grooved cutting tool was proposed by Sugita et al. (2014) to reduce the levels of cutting forces and temperature during bone cutting. Liao et al. (2017) developed a milling cutter with a main cutting edge and micro-cutting edges that diminished significantly the fracture damage of the cut surface in bones as well as the cutting temperature.

Besides, novel bone machining processes were proposed to control fracture/damage and reduce forces, employing ultrasonic vibration (Alam et al., 2011), laser (Wallace et al., 2004) and water jet (Schwieger et al., 2004). Ultrasonically assisted machining of bone basically utilizes high-frequency impacts to change a character of tool-tissue interaction (e.g. piezosurgery). Thanks to its miniaturized device and multiple benefits, it is favored by doctors and researchers. Ultrasonic cutting was adopted to reduce the cutting forces (Ying et al., 2020) and thermal damage (Cardoni et al., 2006) of bone. Alam et al. (2013) performed plane cutting of cortical bone with ultrasonic vibration and investigated the effect of different vibration parameters on the cutting force. However, the microstructure of cortical bone was not considered. Recently, Sugita et al. (2017) and Shu and Sugita (2020) analyzed the fracture phenomenon with a discontinuous bone model in vibration-assisted cutting by employing the extended finite-element approach, incorporating the detailed bone microstructure. Still, the mechanism of chip removal in impact cutting was not investigated by considering the microstructure and anisotropy of cortical bone.

In this study, comparisons of chip morphologies and cutting-induced forces in conventional and high-frequency impact cutting of cortical bone are investigated. The reasons for material-removal improvement and fracture-toughness variation in impact cutting are analyzed.

\section{Materials, methods and experiments}

\subsection{Characteristics of cortical bone}

Cortical bone is a dense and rigid outer layer of bones (Cowin, 2001).
It mainly consists of osteons, interstitial matrix and cement lines as shown in Fig. 1(a). The osteon is the basic unit of the cortical bone; it is regarded as cylinder with diameter of approx. 100-200 $\mu \mathrm{m}$ (Rauch et al., 2007; Cowin, 2001; Ascenzi and Bonucci, 1968). However, the elastic modulus and fracture strength of cement lines are considered by many researchers to be lower than those of osteons and interstitial matrix (Budyn and Hoc, 2007; Abdel-Wahab et al., 2012; Sugita et al., 2017), thus affecting the crack propagation during cutting. Furthermore, the osteon is composed of bone lamellae and a Haversian canal. Therefore, cortical bone is a brittle and rigid composite material with a multi-scale structure. As a result, characteristics of its removal in cutting should be closely related to its microscopic and submicroscopic structure.

\subsection{Sample preparation}

The cortical-bone samples were taken from a fresh bovine femur of the age of 2-3 years in cuboid shape along its circumference, axial and radial directions, so as to obtain samples of transverse, parallel and across directions based on the osteon orientation (Fig. 1(b)). The size of the prepared samples was $20 \mathrm{~mm} \times 3 \mathrm{~mm}$ (width) $\times 20 \mathrm{~mm}$. A cutting edge of the tool was parallel to the width of the samples; thus, the cutting width was fixed at $3 \mathrm{~mm}$. Since the osteon orientation resulted in anisotropy of cortical bone and difference in chip formation, comparison tests were conducted in three cutting directions in this study. The cutting diagrams for these directions of cutting are shown in Fig. 1(b), with letters T, P and A denoting transverse, parallel and across cutting directions, respectively.

\subsection{Impact cutting method}

An impact cutting method is proposed to improve the material removal of cortical bone. The schematic diagram of conventional and impact cutting techniques are shown in Fig. 1(c). In impact cutting, the cutting tool is excited with high frequency by a transducer; thus, the shock waves and intermittent tool-chip interaction affect the material 
removal of cortical bone, which is discussed in detail in Section 3.

\subsection{Experimental works}

A developed experimental setup of bone cutting is illustrated in Fig. 1 (d). The system was composed of a vibration-isolated table, a three-axis high-precision motorized stage, a high-speed microscope Keyence VW9000 (maximum camera resolution is $640 \times 480$ pixels, maximum frame rate is $230000 \mathrm{fps}$ ), a Kistler dynamometer Type 9256A1 and a lamphouse. An autonomous one-dimensional high-frequency impact cutting system was employed to perform conventional cutting and impact cutting regimes, changed by a flexible switch. High-frequency vibration of the cutting tool was driven by a piezoelectric transducer, connected to a horn; the assembly was fixed on the three-axis stage at the vibration wave node. To ensure that the system was in a resonant state, the horn made of titanium alloy was optimized, and the shape of the tool was cut directly at its end, with the tool rake and relief angles of $15^{\circ}$ and $5^{\circ}$, respectively; the tool's width was $10 \mathrm{~mm}$.

To observe the processes of chip formation and crack propagation clearly, the cutting speed was set as $5 \mathrm{~mm} / \mathrm{s}$. The depth of cut was chosen as $200 \mu \mathrm{m}$, close to the diameter of osteon, with fractured chips generated in conventional cutting. The vibration frequency of the cutting tool was optimized as $19.5 \mathrm{kHz}$, while the amplitude was set at $0,10,15,20$ and $25 \mu \mathrm{m}$ to investigate the effect of vibration parameters on chip removal in cortical bone.

Chip formation was captured with a high-speed microscope Keyence VW-9000. The lens magnification was 400 times, the shutter speed was $1 / 3000 \mathrm{~s}$, the frame rate was $1000 \mathrm{fps}$ and the frame size was $640 \times 480$ pixels. Although the maximum frame rate of the high-speed camera was $230000 \mathrm{fps}$, i.e. higher than the tool's vibration frequency $(19.5 \mathrm{kHz})$, the resolution of the image was very low when the high frame rate was used. Therefore, only lower levels of the shutter speed and the frame rate could be employed. So, it was impossible to observe the tool displacement and the contact process with the bone material in a single vibration cycle, but it did not affect the observation of the bone removal process in the experiments.

\section{Results and discussion}

In this section, the chip formation in conventional and impact cutting processes are contrasted in Section 3.1; the cutting forces in these two regimes were compared in Section 3.2. To reveal the differences in chip formation and cutting forces in two cutting processes, the analysis of fracture toughness of cortical bone and its micro-structure are presented in Section 3.3. The aim of investigation of cortical bone's fracture toughness was to illustrate the fracture modes in two regimes, helping to explain the fracture propagation in Section 3.1 and force variation in Section 3.2. A further study of fracture toughness of micro-structure was undertaken to reveal the crack behavior at the interface (cement lines), which could elucidate the essential reason of fracture propagation in two cutting processes.

\subsection{Improvements of chip formation in impact cutting}

To investigate the effect of impact cutting on chip formation, controlled experiments in both conventional and impact cutting regimes of cortical bone were performed successively in transverse, parallel and across directions.

A chip-formation process and crack propagation in cortical bone in transverse direction are presented in Fig. 2(a) for two cutting processes. It is obvious that the morphology of bone chips and the propagation of cracks differ significantly in conventional and impact cutting of cortical bone. During conventional cutting, osteons fractured due to bending and shearing forces when the cutting tool moved uniformly along the cutting direction, with fractures propagating obliquely into the workpiece. Meanwhile, since the strength of cement lines is lower than that of
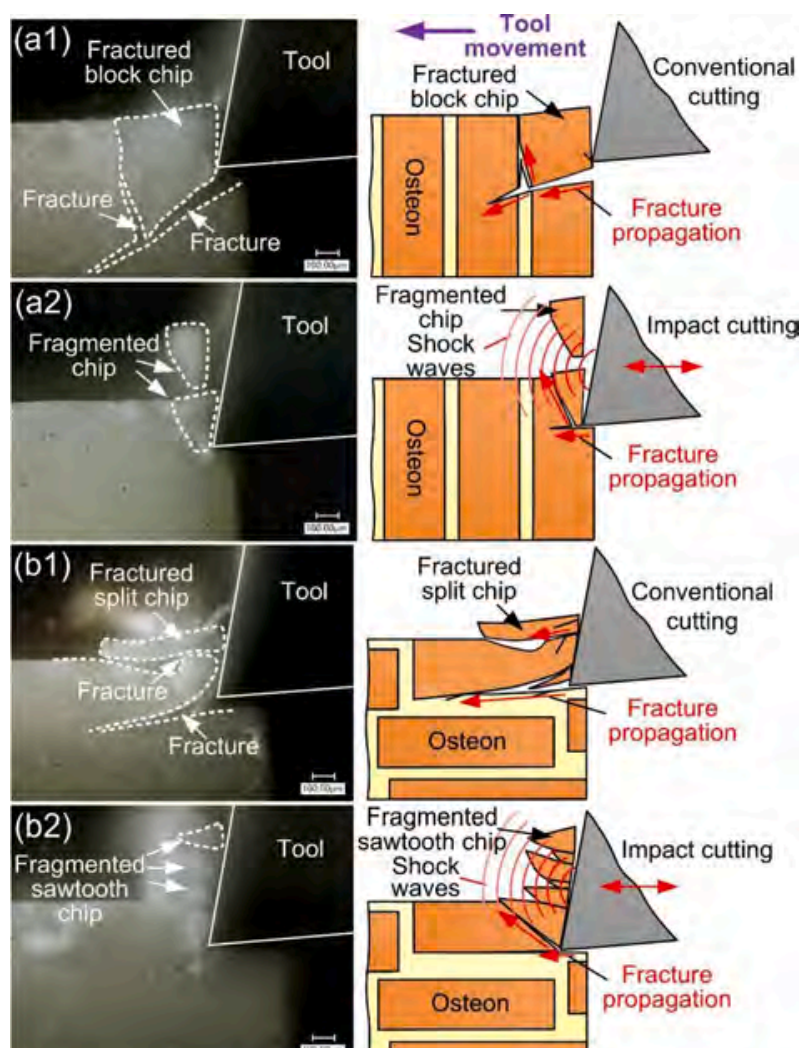

Fragmented
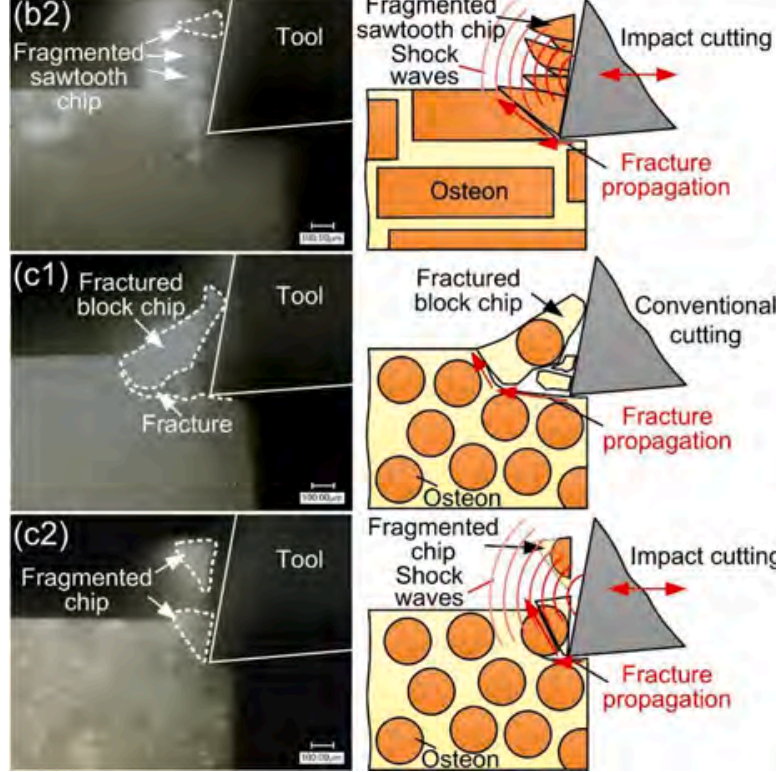

Fragmented

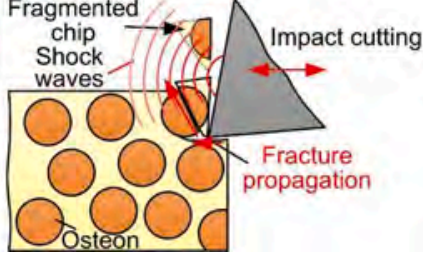

Fig. 2. Chip formation in cortical bone with conventional (1) and impact (2) cutting in transverse (a), parallel (b) and across (c) directions.

osteon (Dong et al., 2005), the fractures propagated along the shear direction in the periphery of the osteon. Thus, fractured block chips were generated (Fig. 2(a1)), where the fracture propagation directions varied and the width of fractures was large. In contrast, the chip morphology of cortical bone in high-frequency impact cutting (Fig. 2(a2)) was apparently different from that in the conventional technique. Under a high-frequency vibratory shock load, the fracture propagation direction changed to nearly parallel to the cutting direction. In addition, some fractures appeared along the main shear direction, which crossed the osteons; thus, the bone material was removed with triangular fragmented chips. Meanwhile, the peeled chips instantly broke away from the rake face of the cutting tool thanks to shock waves. Therefore, impact cutting could generate a new chip-formation mechanism and change both the chip morphology and the fracture propagation character in the transverse-direction cutting.

Bone samples prepared for the parallel direction were used in conventional and impact cutting, and the respective chip-formation process and crack propagation are presented in Fig. 2(b). Two completely 

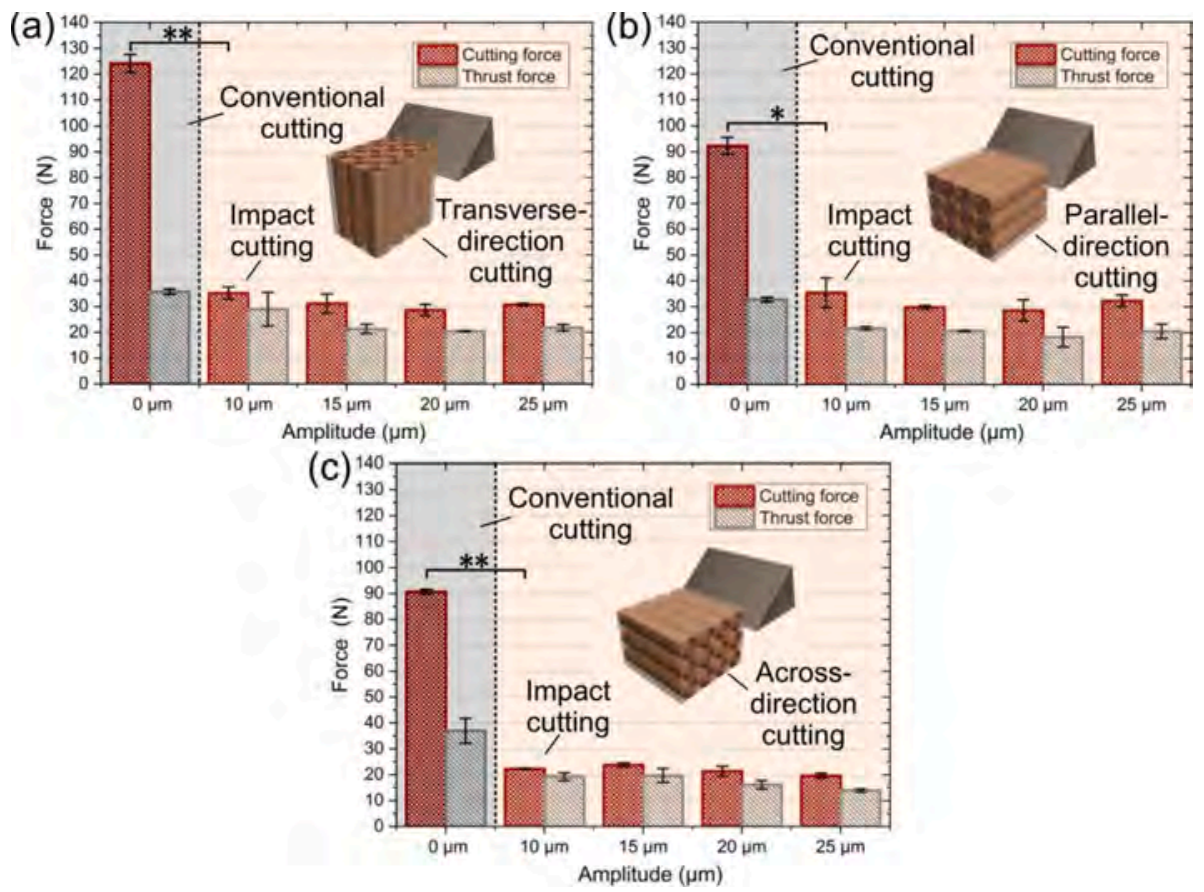

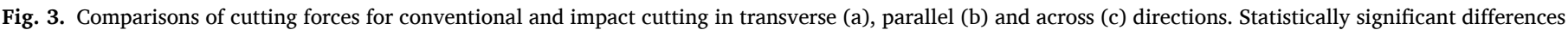
with the corresponding cutting forces in conventional cutting: ${ }^{*} p<0.05,{ }^{* *} p<0.01$.

different chip-formation mechanisms were also generated. In conventional cutting, fractured strip chips were produced, with fractures propagating into the workpiece along the cutting direction, with a very noticeable width. The reason for this behaviour was the orientation of the osteonal axis parallel to the cutting direction. So, during the tool's movement forward with a constant velocity, fractures mainly propagated along the periphery of the osteons as cement lines were weaker than osteons. Meanwhile, the pushing force by the cutting tool caused the internal slip of lamellae in osteons, separating them from each other and resulting in large fractured strip chips (Fig. 2(b1)). In impact cutting, bone chips transformed entirely different, serrated morphology (Fig. 2(b2)). Evidently, high-frequency micro-impacts changed the original mode of fracture. Cracks mainly propagated along the shear direction; thus, fragmented sawtooth chips were formed when the shearremoved parts of osteons and bone matrix formed them. Obviously, an extent of damage of the machined bone surface and fractures in highfrequency impact cutting with shock waves in parallel direction were less than those in conventional cutting.

In the across-direction cutting, the chip-formation process and crack propagation of cortical bone were also studied for two cutting methods (Fig. 2(c)), with similar processes observed, as for the other two directions. In conventional cutting, fracture propagated along the periphery of osteons, with the bone tissue removed in the main shear direction, producing a fractured block chip (Fig. 2(c1)). In impact cutting, the fracture propagated directly through the osteons in the main shear direction, generating triangular fragmented chips (Fig. 2(c2)). Besides, peeled chips broke away instantly from the rake face of the cutting tool by shock waves.

The above experiments for three cutting directions demonstrated that the mechanism of fracture propagation and chip morphology were completely different in conventional and high-frequency impact cutting processes of cortical bone. These differences were mainly manifested in the fracture propagation directly through osteons or interstitial matrix along the main shear direction during high-frequency impact cutting rather than deflection around osteons as a result of different strengths of bone constituents. The main reason was higher strain rates in impact cutting (Bai et al., 2017; Zimmermann et al., 2014), which may change the local bone toughness.

\subsection{Reduction of cutting forces in impact cutting}

Cutting forces in the bone machining process directly affect mechanical damage of the bone tissue as well as the load applied to handheld devices used by doctors in orthopaedic surgeries. Here, cutting forces are compared for cortical bone in conventional and impact cutting with the same cutting parameters (see Section 2.4). The paired $t$ tests were performed for multiple comparisons to determine differences between two regimes in the cutting forces. $p$-Values less than 0.05 and 0.01 are considered statistically significant and extremely significant, respectively. To investigate the effect of impact amplitude on cutting forces, various vibration amplitudes were set as $0,10,15,20$ and $25 \mu \mathrm{m}$, with $0 \mu \mathrm{m}$ representing the conventional cutting process. The (main) cutting forces for cortical bone were greater than the thrust forces under various processing parameters; especially, the difference was highest in conventional cutting (Fig. 3). Besides, the (main) cutting forces in the transverse direction were larger than those in the parallel and across directions in conventional cutting. Apparently, the (main) cutting forces reduced significantly in impact cutting. For a case with the amplitude of $10 \mu \mathrm{m}$ in the transverse direction, the reduction of the (main) cutting force was $71.7 \%(p<0.01)$. Similar declines of this force were obtained for the parallel and across directions $-61.6 \%(p<0.05)$ and $75.4 \%(p<$ 0.01 ), respectively - under the corresponding parameters. Obviously, the reduction of (main) cutting forces in impact cutting with three cutting directions have statistical significant.

The significant reduction of forces in high-frequency impact cutting was not only due to the periodic separation of the tool and the bone workpiece in the cutting process, but also as a result of the change of the fracture-propagation mode caused by a high-frequency-vibration shock, discussed in Section 3.3. Besides, no obvious decreasing trends were found for the increased amplitude. The reason is that the critical cutting speed $(2 \pi f a)$ for ultrasonically assisted cutting at the lowest vibration amplitude $(10 \mu \mathrm{m})$ of the experiments was $1224.6 \mathrm{~mm} / \mathrm{s}$, i.e. much higher than the current cutting speed of $5 \mathrm{~mm} / \mathrm{s}$. Therefore, there was no obvious change in the character of force reduction with increasing 


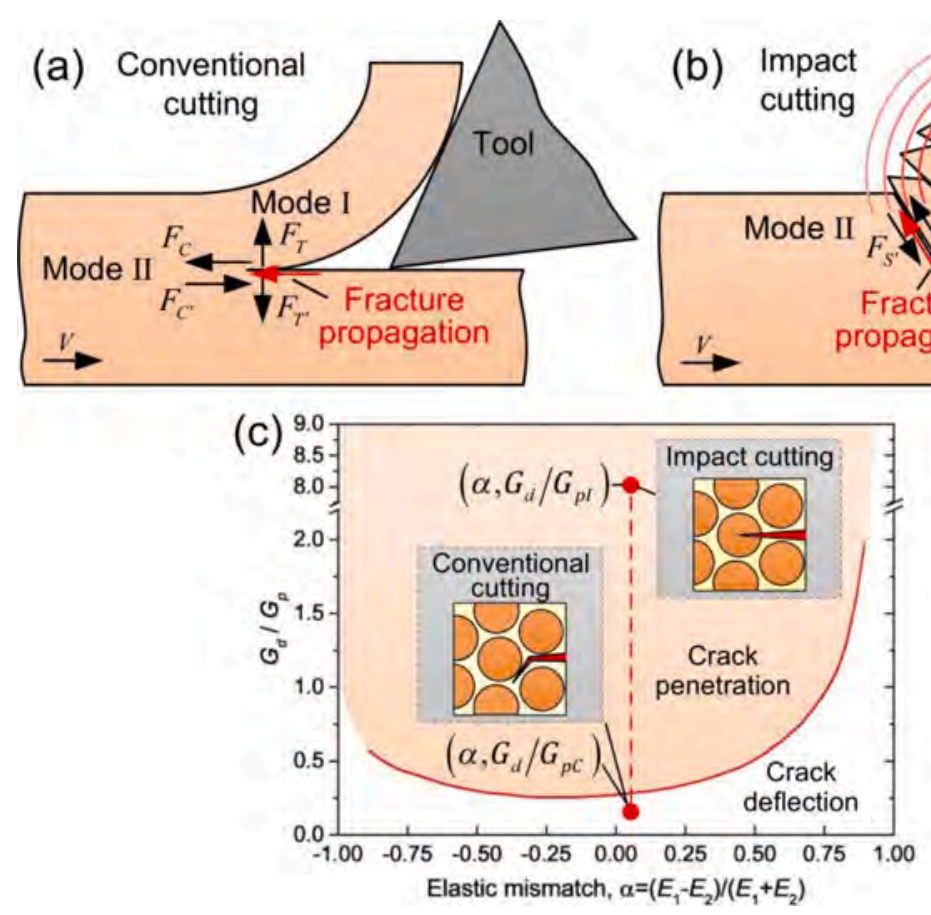

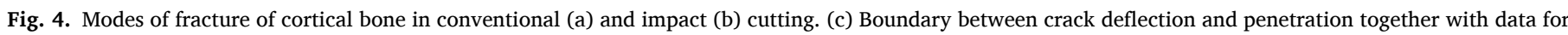
conventional and impact cutting.

amplitude.

\subsection{Analysis of fracture toughness in impact cutting}

To elucidate the improvements of chip formation and reduction of cutting forces in impact cutting, fracture toughness of cortical bone was investigated.

A process of chip formation in bone cutting was divided by Liao and Axinte (2016) into three modes-shear cutting mode, shear-crack mode and fracture cutting mode. These modes were driven by different fracture mechanisms due to mechanical properties of the osteonal structure. Fracture resistance of cortical bone is usually measured in terms of the energy release rate $G$, with the fracture occurring when it exceeds its critical value $G_{C}$.

In conventional cutting with a large depth-of-cut $(200 \mu \mathrm{m})$, fractured chips were generated in the fracture cutting mode. Large cutting $\left(F_{C}\right)$ and thrust $\left(F_{T}\right)$ forces in the cutting and depth-of-cut directions produced the fracture of mode II (shearing) and mode I (opening) (Fig. 4 (a)); thus, the energy release rate of this mix-mode fracture can be calculated as

Table 1

Energy released rate for different fracture directions under conventional and impact cutting.

\begin{tabular}{|c|c|c|c|c|}
\hline & \multicolumn{2}{|c|}{ Conventional cutting } & \multicolumn{2}{|c|}{ Impact cutting } \\
\hline & $\begin{array}{l}\text { Energy } \\
\text { release rate } \\
G_{C, i}\left(10^{3} \mathrm{~N} /\right. \\
\mathrm{m})\end{array}$ & $\begin{array}{l}\text { Critical energy } \\
\text { release rate } \\
G_{(I+I I) C, i}\left(10^{3}\right. \\
\mathrm{N} / \mathrm{m})\end{array}$ & $\begin{array}{l}\text { Energy } \\
\text { release rate } \\
G_{I, i}\left(10^{3} \mathrm{~N} /\right. \\
\mathrm{m})\end{array}$ & $\begin{array}{l}\text { Critical energy } \\
\text { release rate } \\
G_{I I C, i}\left(10^{3} \mathrm{~N} /\right. \\
\mathrm{m})\end{array}$ \\
\hline $\begin{array}{r}\text { Transverse } \\
\text { direction }\end{array}$ & 41 & 16 & 5.8 & 4.7 \\
\hline $\begin{array}{l}\text { Parallel } \\
\text { direction }\end{array}$ & 34 & 2.4 & 6.1 & 2.4 \\
\hline $\begin{array}{l}\text { Across } \\
\text { direction }\end{array}$ & 31 & 9.2 & 3.7 & 3.6 \\
\hline
\end{tabular}

$G_{C, i}=\frac{\partial W}{\partial A}=\frac{\left(\cos \left(\lambda_{i}-\gamma\right)+\sin \left(\lambda_{i}-\gamma\right) \tan \phi_{i}\right)\left(\tau_{s, i} h-G_{I I C, i} \sin \phi_{i}\right)}{\sin \phi_{i} \cos \left(\phi_{i}+\lambda_{i}-\gamma\right)}$,

where $W$ is the released energy calculated from the work of cutting and thrust forces, $A$ is the deformation area. $i=1,2$ and 3 denote cutting in transverse, parallel and across directions, respectively. $\lambda, \gamma$ and $\phi$ are the nominal friction angle, the rake angle of the tool and the nominal shear angle, respectively. $\tau_{s}$ is the shear stress, $h$ is the depth of cut, $G_{I I C}$ is the critical energy release rate under mode-II fracture.

However, in impact cutting, fragmented sawtooth chips were generated, where the fracture of mode II was induced by the shear force $\left(F_{S}\right)$ in the main shear plane under high-frequency impact (Fig. 4(b)). The energy release rate in this plane can be expressed as

$G_{I, i}=\frac{\partial W_{s}}{\partial A_{s}}=\frac{\tau_{s, i} h}{\sin \phi_{i}}$

where $W_{s}$ is the released energy obtained from the work of shear force, $A_{s}$ is the area of shear deformation.

The nominal friction coefficient $(\mu)$ and shear stress $\left(\tau_{s}\right)$ can be evaluated for bone cutting in three directions from the experimental results as

$\mu_{i}=\frac{F_{C, i} \tan \gamma+F_{T, i}}{F_{C, i}-F_{T, i} \tan \gamma}$,

$\tau_{s, i}=\frac{F_{C, i} \cos \phi_{i}-F_{T, i} \sin \phi_{i}}{w h / \sin \phi_{i}}$,

where $\mu$ can be also expressed as $\tan \lambda, F_{C}$ and $F_{T}$ are the cutting and thrust forces, respectively. Besides, the shear angle $\phi$ was calculated as $\pi / 4-(\lambda-\gamma) / 2$, based on the Merchant's solution (Merchant, 1945).

The energy release rate in the two studied cutting processes for three directions can be calculated from Eqs. (1)-(4). The critical energy release rate of cortical bone for different fracture modes was studied by Feng et al. (2000). In addition, a mixed-mode (I and II) fracture criterion can be evaluated for two loading modes as follows: 
Table 2

Material properties for fracture toughness of bone micro-structures (Sugita et al., 2017; Chan and Nicolella, 2012).

\begin{tabular}{|c|c|c|c|c|c|}
\hline $\begin{array}{l}\text { Elastic modulus } \\
E_{1}(\mathrm{GPa})\end{array}$ & $\begin{array}{l}\text { Elastic modulus } \\
E_{2}(\mathrm{GPa})\end{array}$ & $\begin{array}{l}\text { Dundurs } \\
\text { parameter } \alpha\end{array}$ & $\begin{array}{l}\text { Interface toughness } K_{i} \\
\left(\mathrm{MPa} \cdot \mathrm{m}^{1 / 2}\right)\end{array}$ & $\begin{array}{l}\text { Penetration toughness of osteons in } \\
\text { conventional cutting } K_{p C}\left(\mathrm{MPa} \cdot \mathrm{m}^{1 / 2}\right)\end{array}$ & $\begin{array}{l}\text { Penetration toughness of osteons in } \\
\text { impact cutting } K_{p I}\left(\mathrm{MPa} \cdot \mathrm{m}^{1 / 2}\right)\end{array}$ \\
\hline 22.8 & 20.7 & 0.05 & 0.89 & 2.4 & 0.3 \\
\hline
\end{tabular}

$G_{(I+I I) C, i}=\frac{\left(K_{I C, i}\right)^{2}+\left(K_{I I C, i}\right)^{2}}{E_{i} /\left(1-\nu_{i}^{2}\right)}$

where $K_{I C}$ and $K_{I I C}$ are the fracture toughness (critical stress intensity factor) of cortical bone in loading modes I and II, respectively. $E$ and $\nu$ are the elastic modulus and the Poisson's ratio, respectively. Thus, the energy release rate for different fracture directions under conventional and impact cutting can be obtained (Table 1). Obviously, it can be concluded for each fracture direction:

$G_{C, i}>G_{(I+I I) C, i}, G_{I, i}>G_{I I C, i}$,

which means that the energy release rate in conventional cutting $\left(G_{C, i}\right)$ with above experimental conditions exceeded that for the mixed-mode regime $\left(G_{(I+I I) C, i}\right)$, and that in impact cutting $\left(G_{I, i}\right)$ was higher than the critical value for the shearing mode $\left(G_{I I C, i}\right)$; thus, fractured and fragmented chips were generated, respectively. In addition, an obvious relationship can be found:

$G_{C, i} \gg G_{I, i}$,

indicating that the energy release rate in conventional cutting was considerably larger than that in impact cutting with the same cutting parameters, i.e. less work was needed to remove a unit volume of the bone material in the latter case. Although periodic separation of the tool from the bone workpiece contributed significantly to the reduction of cutting forces in impact cutting, the lower energy release rate led to minimal consumption of the cutting energy when fractures penetrated in the main shear direction, thus reducing macroscopic cutting forces.

To reveal the essential process of crack propagation under impact load, the fracture toughness of micro-structure should be analyzed. During tool-bone interaction, crack deflection at the interface (cement lines) or crack penetration through the osteons is determined by the ratio of the elastic-energy release rates $-G_{d}$ for interface deflection and $G_{p}$ for penetration. Critical conditions for crack deflection and penetration were proposed with a delamination map by $\mathrm{He}$ and Hutchinson (1989), for a crack tip that resides in a region with elastic modulus $E_{1}$ (in our case, interstitial matrix) and extends into another region with elastic modulus $E_{2}$ (osteon). The elastic mismatch between the interstitial and osteonal bone is expressed with Dundurs parameter, defined as

$\alpha=\frac{E_{1}-E_{2}}{E_{1}+E_{2}}$.

Crack deflection at the interface happens when $G_{d} / G_{p}$ is less than the critical value, while penetration occurs when the ratio exceeds it. In impact cutting, due to the effect of higher strain rates on stress intensity, fracture toughness of bone varies. The $G_{d} / G_{p}$ ratio associated with a crack tip is expressed as

$\frac{G_{d}}{G_{p}}=\left\{\begin{array}{c}\frac{G_{d}}{G_{p C}}=\left[\frac{K_{i}}{K_{p C}}\right]^{2}\left[\frac{E_{2}}{E_{1}}\right], \text { for conventional cutting } \\ \frac{G_{d}}{G_{p I}}=\left[\frac{K_{i}}{K_{p I}}\right]^{2}\left[\frac{E_{2}}{E_{1}}\right], \text { for impact cutting }\end{array}\right.$

where $K_{i}$ is the interface toughness between the interstitial matrix and the osteon, $G_{p C}$ and $K_{p C}$ are the elastic-energy release rate and fracture toughness for crack penetration into osteon in conventional cutting, $G_{p I}$ and $K_{p I}$ are these parameters for impact cutting.

Fracture toughness was evaluated from a load-displacement diagram in dynamic testing with a modified Split-Hopkinson bar for strain rate between $10^{-3}$ and $10^{3} \mathrm{~s}^{-1}$, and the stress intensity rate varied between $\sim_{10^{-3}}$ and $10^{5} \mathrm{MPa} \cdot \mathrm{m}^{1 / 2} \cdot \mathrm{s}^{-1}$ (Adharapurapu et al., 2006). The stress intensity factor $K$ was evaluated as a function of time, with the fracture time $t_{f}$ yielding the fracture toughness. Based on high-speed videos of chip formation in the experiments and the time-force data, the fracture times for cortical bone in conventional (quasi-static loading rate) $t_{f C}$ and impact (high stress intensity loading rate) $t_{\text {fI }}$ cutting were calculated with average values of $36 \mu$ s and $12 \mu \mathrm{s}$, respectively. Therefore, the fracture toughness of cortical bone for penetration in conventional $\left(K_{p C}\right)$ and impact $\left(K_{p I}\right)$ cutting were estimated as 2.4 and $0.3 \mathrm{MPa} \cdot \mathrm{m}^{1 / 2}$, respectively. Obviously, high-speed impacts reduced the fracture toughness of the machined cortical bone significantly.

The interface (cement lines) toughness $K_{i}$ was assumed unchanged in different conditions (Chan et al., 2009). The material properties used for fracture toughness are shown in Table 2. So, the ratios $G_{d} / G_{p C}$ and $G_{d} /$ $G_{p I}$ were obtained as 0.12 and 7.99. A delamination map, shown in Fig. 4 (c), demonstrates a boundary between crack deflection and penetration based on Chan's data (Chan and Nicolella, 2012), with the two points of toughness status calculated for two strain rates corresponding to conventional and impact cutting - $\left(\alpha, G_{d} / G_{p C}\right)$ and $\left(\alpha, G_{d} / G_{p I}\right)$ - plotted. Apparently, the point for low strain rates (conventional cutting) lies below the critical condition, with cracks deflecting at the interface (cement lines), while the point is above this boundary at high strain rates (impact cutting), i.e. cracks penetrate the osteons. Besides, the location of point $\left(\alpha, G_{d} / G_{p I}\right)$ demonstrates that the degree of variation of fracture toughness and crack extension in impact cutting are much higher than those in conventional cutting, and even higher than in most high-strain-rate toughness tests of bone.

\section{Conclusions}

This paper studied a high-frequency impact cutting method for improving chip removal of cortical bone. Experimental results indicated that fracture propagated directly through the osteons or interstitial matrix along the main shear direction in impact cutting rather than being deflected along cement lines as in conventional cutting. This impact method generated fragmented chips instead of fractured blocks or split chips observed in conventional cutting and reduced the level of cutting forces significantly at various impact amplitudes. A lower energy release rate led to the lower consumption of the cutting energy when cutting-generated cracks propagated in the main shear direction in the impact process, with the resulting reduced macroscopic cutting forces being an important reason. Chip morphologies changed because the higher strain rates in high-frequency impact cutting led to reduction of bone toughness, resulting in fracture development in the osteonal matrix along a straighter path rather than deflecting and following the cement lines at lower strain rates. These conclusions have important theoretical and practical significance for developing innovative orthopaedic instruments.

In addition, a temperature rise in high-frequency impact cutting could generate thermal damage, although the nominal cutting speed ( 5 $\mathrm{mm} / \mathrm{s}$ ) used in the experiments was far below the critical speed for thermal necrosis (Alam et al., 2010), while the maximum cutting speed was very high. So, thermal analysis in impact cutting will be studied further. 


\section{Declaration of competing interest}

The authors declare no conflict of interest.

\section{CRediT authorship contribution statement}

Wei Bai: Methodology, Investigation, Writing - original draft. Liming Shu: Data curation. Ronglei Sun: Visualization. Jianfeng Xu: Supervision. Vadim V. Silberschmidt: Supervision. Naohiko Sugita: Conceptualization.

\section{Acknowledgement}

This work was supported by the China Postdoctoral Science Foundation through grants No. 2019M652629 and No. 2019TQ0107. The authors gratefully acknowledge the support provided by Department of Mechanical Engineering, School of Engineering, The University of Tokyo for this work.

\section{References}

Abdel-Wahab, A.A., Maligno, A.R., Silberschmidt, V.V., 2012. Micro-scale modelling of bovine cortical bone fracture: analysis of crack propagation and microstructure using X-FEM. Comput. Mater. Sci. 52, 128-135.

Adharapurapu, R.R., Jiang, F., Vecchio, K.S., 2006. Dynamic fracture of bovine bone. Mater. Sci. Eng. C 26, 1325-1332.

Alam, K., Khan, M., Silberschmidt, V.V., 2013. Analysis of forces in conventional and ultrasonically assisted plane cutting of cortical bone. Proc. Inst. Mech. Eng. H 227, 636-642.

Alam, K., Mitrofanov, A., Silberschmidt, V.V., 2010. Thermal analysis of orthogonal cutting of cortical bone using finite element simulations. Int. J. Exp. Comput. BioMech. 1, 236-251.

Alam, K., Mitrofanov, A.V., Silberschmidt, V.V., 2011. Experimental investigations of forces and torque in conventional and ultrasonically-assisted drilling of cortical bone. Med. Eng. Phys. 33, 234-239.

Ascenzi, A., Bonucci, E., 1968. The compressive properties of single osteons. Anat. Rec. 161, 377-391.

Bai, W., Shu, L., Sun, R., Xu, J., Silberschmidt, V.V., Sugita, N., 2020. Mechanism of material removal in orthogonal cutting of cortical bone. J. Mech. Behav. Biomed. $104,, 103618$

Bai, W., Sun, R., Leopold, J., Silberschmidt, V.V., 2017. Microstructural evolution of Ti6Al4V in ultrasonically assisted cutting: numerical modelling and experimenta analysis. Ultrasonics 78, 70-82.
Budyn, E., Hoc, T., 2007. Multiple scale modeling of cortical bone fracture in tension using X-FEM. Europ. J. Comput. Mech. 16, 213-236.

Cardoni, A., MacBeath, A., Lucas, M., 2006. Methods for reducing cutting temperature in ultrasonic cutting of bone. Ultrasonics 44, 37-42.

Chan, K.S., Chan, C.K., Nicolella, D.P., 2009. Relating crack-tip deformation to mineralization and fracture resistance in human femur cortical bone. Bone 45, 427-434.

Chan, K.S., Nicolella, D.P., 2012. Micromechanical modeling of R-curve behaviors in human cortical bone. J. Mech. Behav. Biomed. 16, 136-152.

Cowin, S.C., 2001. Bone Mechanics Handbook. CRC Press.

Dong, X.N., Zhang, X., Guo, X.E., 2005. Interfacial strength of cement lines in human cortical bone. Mech. Chem. Biosyst. 2, 63-68.

Feldmann, A., Ganser, P., Nolte, L., Zysset, P., 2017. Orthogonal cutting of cortical bone: temperature elevation and fracture toughness. Int. J. Mach. Tool Manufact. 118 $1-11$.

Feng, Z., Rho, J., Han, S., Ziv, I., 2000. Orientation and loading condition dependence of fracture toughness in cortical bone. Mater. Sci. Eng. C 11, 41-46.

He, M.Y., Hutchinson, J.W., 1989. Crack deflection at an interface between dissimilar elastic materials. Int. J. Solid Struct. 25, 1053-1067.

Jacobs, C.H., Pope, M.H., Berry, J.T., Hoaglund, F., 1974. A study of the bone machining process - orthogonal cutting. J. Biomech. 7, 131-136.

Liao, Z., Axinte, D.A., 2016. On chip formation mechanism in orthogonal cutting of bone. Int. J. Mach. Tool Manufact. 102, 41-55.

Liao, Z., Axinte, D.A., Gao, D., 2017. A novel cutting tool design to avoid surface damage in bone machining. Int. J. Mach. Tool Manufact. 116, 52-59.

Merchant, M.E., 1945. Mechanics of the metal cutting process. II. Plasticity conditions in orthogonal cutting. J. Appl. Phys. 16, 318-324.

Rauch, F., Travers, R., Glorieux, F.H., 2007. Intracortical remodeling during human bone development-a histomorphometric study. Bone 40, 274-280.

Schwieger, K., Carrero, V., Rentzsch, R., Becker, A., Bishop, N., Hille, E., Louis, H., Morlock, M., Honl, M., 2004. Abrasive water jet cutting as a new procedure for cutting cancellous bone-in vitro testing in comparison with the oscillating saw. J. Biomed. Mater. Res. B 71, 223-228.

Shu, L., Sugita, N., 2020. Analysis of fracture, force, and temperature in orthogonal elliptical vibration-assisted bone cutting. J. Mech. Behav. Biomed. 103, 103599.

Sugita, N., Ishii, K., Sui, J., Terashima, M., 2014. Multi-grooved cutting tool to reduce cutting force and temperature during bone machining. CIRP Ann. - Manuf. Technol. 63, 101-104.

Sugita, N., Mitsuishi, M., 2009. Specifications for machining the bovine cortical bone in relation to its microstructure. J. Biomech. 42, 2826-2829.

Sugita, N., Shu, L., Shimada, T., Oshima, M., Kizaki, T., Mitsuishi, M., 2017. Novel surgical machining via an impact cutting method based on fracture analysis with a discontinuum bone model. CIRP Ann. - Manuf. Technol. 66, 65-68.

Wallace, R.J., Whitters, C.J., McGeough, J.A., Muir, A., 2004. Experimental evaluation of laser cutting of bone. J. Mater. Process. Technol. 149, 557-560.

Ying, Z., Shu, L., Sugita, N., 2020. Experimental and finite element analysis of force and temperature in ultrasonic vibration assisted bone cutting. Ann. Biomed. Eng. 1-10.

Zimmermann, E.A., Gludovatz, B., Schaible, E., Busse, B., Ritchie, R.O., 2014. Fracture resistance of human cortical bone across multiple length-scales at physiological strain rates. Biomaterials 35, 5472-5481. 